\title{
9 Autorschaft
}

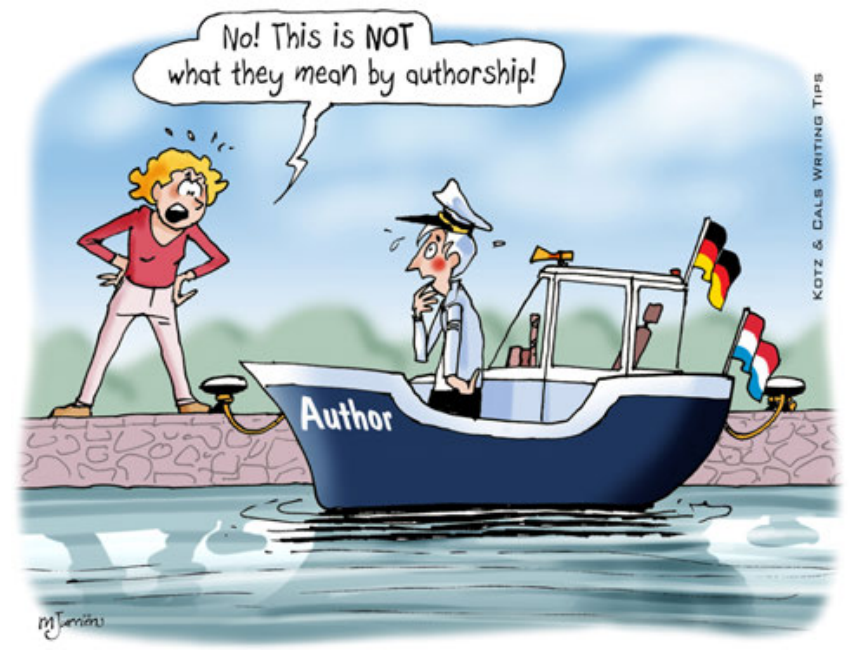

Tipp 9 - Autorenschaft: diskutiere dies frühzeitig mit dem Projektteam!

\section{Was Sie wissen sollten}

Für Ihre wissenschaftliche Laufbahn ist es wichtig, Autor wissenschaftlicher Artikel zu sein und dabei eine Schlüsselposition zu haben (vor allem als Erst- oder Letztautor). Deswegen überrascht es nicht, dass Autorschaft ein viel diskutiertes Thema in Besprechungsräumen und an den Kaffeemaschinen akademischer Abteilungen ist. Um Autor eines Artikels zu sein müssen laut International Committee of Medical Journal Editors (ICMJE, www.icmje.org) drei Kriterien erfüllt sein (siehe Checkliste). Mitwirkende an einem Forschungsprojekt, die nicht diese Kriterien für Autorschaft erfüllen, können (mit deren Erlaubnis) in der Danksagung aufgelistet werden, vorzugsweise mit einer kurzen Beschreibung, welchen Beitrag sie geleistet haben. Autoren werden normalerweise gebeten, Formulare für Autorschaft und Interessenkonflikte zu unterzeichnen. In großen, multizentrischen Studien kann eine Gruppenautorschaft gewählt werden, bei der die wichtigsten Forscher als Autoren aufgelistet werden, gefolgt von „im Namen der XYZ-Gruppe“. Die Mitglieder dieser Gruppe werden in dem Artikel dann nur in der Danksagung aufgelistet, jedoch in Suchmaschinen wie PubMed meist als Autoren identifiziert. 
In biomedizinischen Forschungsbereichen vieler Länder ist die wichtigste Position auf einem Artikel die des ersten Autors, gefolgt vom letzten Autor und dem zweiten Autor. Einige Zeitschriften erlauben eine geteilte Erstautorschaft der ersten beiden Autoren, die entsprechend gekennzeichnet wird. Einer der Autoren auf einem Artikel muss immer als Korrespondenzautor identifiziert werden und ist während des Herausgabeprozesses und nach der Veröffentlichung die Hauptansprechperson für Fragen in Verbindung mit der zugrunde liegenden Arbeit. Immer mehr Zeitschriften bitten zudem, dass einer der Autoren als Bürge (,guarantor“) identifiziert wird und damit die Verantwortung für die Integrität der Arbeit übernimmt, vom Beginn des Forschungsprojekts bis zum veröffentlichten Artikel. Oftmals sind der Korrespondenzautor und/oder der Bürge der Erstautor auf einem Artikel (meist die Person, die das Projekt durchgeführt hat) oder der Letztautor (meist der Projektleiter).

\section{Was Sie tun sollten}

Besprechen Sie gegenseitige Erwartungen in Bezug auf Mitarbeit in einem Forschungsprojekt und Autorschaft zu Projektbeginn und evaluieren Sie diese von Zeit zu Zeit. Aus den meisten Projekten entstehen mehrere Artikel, und die Autorenrollen können bei jedem Artikel anders sein. Über die Erstautorenschaft sollte bereits vor dem ersten Entwurf eines Artikels im Projektteam Einigkeit bestehen, da der Erstautor eine prominente Rolle hat und den Schreibprozess leitet (siehe Teil 1: Anfangen zu Schreiben). Denken Sie daran, dass sich Mitarbeit und Autorenschaft ändern können, z. B. weil Koautoren eine andere Laufbahn einschlagen, an andere Orte umziehen oder das Interesse an Ihrem Forschungsprojekt verlieren können.

Es ist hilfreich, ein Kernteam von höchstens 2-3 Personen zu haben, die typischerweise die täglichen Entscheidungen in einem Projekt treffen und die auch die Rangfolge der Autorschaft besprechen. Ziehen Sie es in Erwägung, die Vereinbarungen in Bezug auf Mitarbeit in einem Forschungsprojekt und Autorschaft schriftlich festzulegen, wobei klar sein sollte, dass sich diese im Laufe des Projekts ändern und neu verhandelt werden können. Das Aufsetzen einer schriftlichen Vereinbarung zwingt ein Team dazu, zu besprechen, was eine Autorschaft rechtfertigt, und welche Faktoren eine Autorschaft oder die Rangfolge der Autoren während des Projekts beeinflussen könnten. Die Autorschaft auf einem Artikel ist eine Anerkennung der eine Leistung gegenüberstehen sollte.

Seien Sie sich als leitender (erster) Autor bewusst, dass die Zusammenarbeit mit mehreren Koautoren eine gute Planung erfordert. Überlegen Sie sich, mit welchem Koautor Sie am besten zusammenarbeiten können. Mit dieser Person 
bereiten Sie am besten den ersten Entwurf Ihres Artikels vor und halten Sie Rücksprache bei schwierigen oder widersprüchlichen Vorschlägen zur Überarbeitung, die Sie von anderen Koautoren erhalten. Wenn Sie einen Entwurf zur kritischen Prüfung an alle Koautoren verteilen, sollten Sie klarmachen, was Sie zum jeweiligen Zeitpunkt von ihnen erwarten: beispielsweise allgemeines Feedback zum inhaltlichen Aufbau oder spezielle Kommentare oder detaillierte Korrekturen zu bestimmten Textabsätzen. Setzen Sie Ihren Koautoren eine Frist zur Beantwortung, und bitten Sie sie, Ihnen mitzuteilen, wenn diese nicht eingehalten werden kann. Sobald ein Artikel Ihren Schreibtisch verlassen hat, können Sie nicht daran weiterarbeiten. Daher kann die Planung Ihres Projekts als Ganzes sowie einzelner Artikel im Besonderen durch einen nicht reagierenden Koautor beeinträchtigt werden. Bitten Sie die Koautoren, bei der finalen Version des Artikels ihre Namen einschließlich Initialen, Titel und Affiliationen genau zu überprüfen, da mögliche Fehler von Datenbanken wie PubMed übernommen werden. Erwägen Sie, sich schon am Anfang Ihrer Karriere eine Wissenschaftler-Identifikationsnummer zuzulegen, z. B. über Orcid (www.orcid.org). Dies hilft, einzelne Personen aus der Wissenschaft zu identifizieren und diese mit ihren Arbeiten und Affiliationen zu verbinden, über Forschungsdisziplinen, Grenzen und Zeit hinweg.

Wenn Sie die hier genannten Empfehlungen berücksichtigen sollte das Ihnen helfen, als Autor die gewünschte Position auf einem Artikel zu erhalten, Artikel mit zahlreichen Koautoren zu koordinieren, und sicher zu stellen, dass jeder (einschließlich Ihnen selbst!) die Arbeit erledigt, die eine Autorschaft rechtfertigt. 


\section{Checkliste für die Autorschaft}

- Besprechen Sie die Autorschaft in einer frühen Phase des Projekts und halten Sie entsprechende Vereinbarungen (insbesondere zur Erstautorschaft) fest

- Überprüfen und befolgen Sie die folgenden ICMJE-Kriterien zu Autorschaft: Autoren sollten ...

1. substantiell zur Konzeption und dem Design der Studie, dem Sammeln der Daten, oder der Analyse und Interpretation der Daten beigetragen haben,

2. zum Schreiben des Artikels oder dessen kritischer Prüfung auf wichtigen intellektuellen Inhalt beigetragen haben, und

3. ihre Zustimmung zu der zur Veröffentlichung vorgesehenen Version des Artikels gegeben haben.

- Erstellen Sie eine Dokument zur Autorenschaft, in dem Sie die anvisierten Autoren benennen, sowie Kriterien für Autorenschaft und Faktoren, die eine Autorenschaft beeinflussen (oder deren Reihenfolge) im Verlauf der Zeit beeinflussen

- Bitten Sie Koautoren mittels zielgerichteter Fragen um eine kritische Prüfung und Feedback, und setzen Sie eine Frist zur Beantwortung.

- Erinnern Sie Koautoren daran, ihre Namen, Initialen und Affiliationen genau zu prüfen bevor Sie den Artikel einreichen.

- Erwägen Sie die Verwendung einer Wissenschaftler-Identifikationsnummer und fragen Sie Ihre Koautoren danach, z. B. über www.orcid.org. 